\title{
Reconsidering an important subclass of high-risk dural arteriovenous fistulas for stereotactic radiosurgery
}

\author{
Daniel A. Tonetti, MD, ${ }^{1,2}$ Bradley A. Gross, MD, ${ }^{1,2}$ Brian T. Jankowitz, MD,,2 \\ Hideyuki Kano, MD, PhD, , Edward A. Monaco III, MD, PhD, ${ }^{1,2}$ Ajay Niranjan, MD, ${ }^{1,2}$ \\ John C. Flickinger, MD, ${ }^{1,3}$ and L. Dade Lunsford, MD ${ }^{1,2}$ \\ ${ }^{1}$ Center for Image-Guided Neurosurgery and Departments of ${ }^{2}$ Neurological Surgery and ${ }^{3}$ Radiation Oncology, University of
Pittsburgh Medical Center, Pittsburgh, Pennsylvania
}

OBJECTIVE Aggressive dural arteriovenous fistulas (dAVFs) with cortical venous drainage (CVD) are known for their relatively high risk of recurrent neurological events or hemorrhage. However, recent natural history literature has indicated that nonaggressive dAVFs with CVD have a significantly lower prospective risk of hemorrhage. These nonaggressive dAVFs are typically diagnosed because of symptomatic headache, pulsatile tinnitus, or ocular symptoms, as in low-risk dAVFs. Therefore, the viability of stereotactic radiosurgery (SRS) as a treatment for this lesion subclass should be investigated.

METHODS The authors evaluated their institutional experience with SRS for dAVFs with CVD for the period from 1991 to 2016, assessing angiographic outcomes and posttreatment hemorrhage rates. They subsequently pooled their results with those published in the literature and stratified the results based on the mode of clinical presentation.

RESULTS In an institutional cohort of 42 dAVFs with CVD treated using SRS, there were no complications or hemorrhages after treatment in 19 patients with nonaggressive dAVFs, but there was 1 radiation-induced complication and 1 hemorrhage among the 23 patients with aggressive dAVFs. In pooling these cases with 155 additional cases from the literature, the authors found that the hemorrhage rate after SRS was significantly lower among the patients with nonaggressive dAVFs ( $0 \%$ vs $6.8 \%, p=0.003)$. Similarly, the number of radiation-related complications was 0/124 in nonaggressive dAVF cases versus $6 / 73$ in aggressive dAVF cases $(p=0.001)$. The annual rate of hemorrhage after SRS for aggressive fistulas was $3.0 \%$ over 164.5 patient-years, whereas none of the nonaggressive fistulas bled after radiosurgery over 279.4 patient-years of follow-up despite the presence of CVD.

CONCLUSIONS Cortical venous drainage is thought to be a significant risk factor in all dAVFs. In the institutional experience described here, SRS proved to be a low-risk strategy associated with a very low risk of subsequent hemorrhage or radiation-related complications in nonaggressive dAVFs with CVD.

https://thejns.org/doi/abs/10.3171/2017.10.JNS171802

KEY WORDS dural arteriovenous fistula; dAVF; SRS; hemorrhage; vascular disorders; stereotactic radiosurgery

$\mathrm{D}$ URAL arteriovenous fistulas (dAVFs) with cortical venous drainage (CVD) are considered high risk because of the attendant risk of venous hypertension associated with both nonhemorrhagic neurological deficits (NHNDs) and frank intracranial hemorrhage. ${ }^{1}$ Embolization is often the first-line treatment for these lesions, with surgery often serving as second-line therapy when the fistula site cannot be occluded or reached with embolisate. ${ }^{3,9,11,13,17}$ A considerable body of evidence il- lustrates the feasibility and success of stereotactic radiosurgery (SRS) for dAVFs as well.,12,18,21 Unfortunately, most reports pool results for high- and low-risk dAVFs and evaluate only angiographic obliteration as the primary outcome. The most relevant issue for dAVFs with CVD is protection from NHNDs and hemorrhages, and this issue is less frequently addressed; however, most would posit that such dAVFs ought to be treated via embolization or surgical disconnection of the CVD.

ABBREVIATIONS CVD = cortical venous drainage; $\mathrm{dAVF}=$ dural arteriovenous malformation; NHND = nonhemorrhagic neurological deficit; $\mathrm{SRS}=$ stereotactic radiosurgery.

SUBMITTED July 23, 2017. ACCEPTED October 3, 2017.

INCLUDE WHEN CITING Published online March 16, 2018; DOI: 10.3171/2017.10.JNS171802. 
Accumulating natural history data suggest that outcome data for dAVFs with CVD should be stratified by "nonaggressive" and "aggressive" presenting symptoms, the latter more specifically referring to those patients who had sustained an NHND or hemorrhage. ${ }^{20}$ It is clear that an evaluation of SRS for these dAVFs ought to be stratified as well. Natural history studies have suggested that there is a significantly lower risk of hemorrhage from nonaggressive dAVFs with CVD. ${ }^{10,19,20}$ We sought to determine whether SRS was an appropriate treatment for these lesions, with an anticipated lower risk of clinical events or hemorrhage during the latency interval. In this report, we evaluate our institutional experience with dAVFs with CVD treated using SRS, present our overall results, and stratify the results based on whether the patient had an aggressive or nonaggressive dAVF. These results were subsequently pooled with additional outcome data extracted from the literature.

\section{Methods}

We reviewed our institution's prospectively collected Gamma Knife database for dAVFs treated with SRS in the period from January 1991 through December 2016. Pretreatment angiograms were reviewed to determine if dAVFs did or did not have CVD. Patient age, sex, mode of clinical presentation, dAVF location, prior embolization, margin dose, target volume, procedural complications, and clinical and radiographic follow-ups were extracted from the medical record.

Stereotactic radiosurgery was performed with the model U, B, C, 4C, or Perfexion Leksell Gamma Knife (Elekta AB). Each patient underwent placement of a stereotactic model $\mathrm{G}$ Leksell frame while under conscious sedation. Target acquisition was obtained using highresolution MRI, followed immediately by high-resolution digital subtraction angiography. All images were exported to a computer workstation for dose planning. The mean margin SRS dose was 21 Gy (median 20 Gy, range 15-25 Gy), and the mean maximal dose was 41 Gy (median 40 Gy, range 30-50 Gy). The mean target volume was 2.65 $\mathrm{cm}^{3}$ (median $2.05 \mathrm{~cm}^{3}$, range $0.46-9.8 \mathrm{~cm}^{3}$ ). Patients underwent repeat clinical evaluation with MRI at 6 months and 1 year after SRS, and angiography was performed at 24-36 months posttreatment.

For our pooled literature analysis, the PubMed database was queried using the terms "stereotactic," "radiosurgery," "dural arteriovenous fistula," and "dAVF." References within selected articles were also perused and, if appropriate, were incorporated for completeness. We only included in our analysis those articles that provided clinical results for the risk of hemorrhage or NHND after treatment specifically for dAVFs with CVD. Studies that did not provide these results or those that provided only overall results for all dAVFs with and without CVD were excluded. Case reports and duplicate data sets were also excluded. From these studies, we extracted presentation mode, treatment complications, follow-up duration, and reported posttreatment NHND events and hemorrhages. These results were then pooled with our own. Comparisons between the cohorts for posttreatment complications and hemorrhages were performed using the Fisher exact test. A p value $\leq 0.05$ was accepted as significant.

\section{Results \\ Institutional Cohort Review}

Sixty-one patients with dAVFs were treated with SRS over a 25-year interval at our institution. Nineteen patients with dAVFs without CVD were excluded; therefore, 42 (69\%) of the $61 \mathrm{dAVF}$ patients had CVD during the reviewed study period. The mean patient age was 61 years old (range 12-90 years), and 60\% of the patients were male. Thirteen patients $(31 \%)$ presented with intracranial hemorrhage, and $11(26 \%)$ had NHND. Among the remaining 19 patients, 7 (17\%) had ocular symptoms, 7 $(17 \%)$ had headaches, and $12(29 \%)$ had pulsatile tinnitus.

Thirteen fistulas (31\%) were located along the tentorium and/or involved the petrosal sinuses, 9 (21\%) were located along the transverse and/or sigmoid sinus, 7 (17\%) were cavernous, 7 (17\%) were located along the superior sagittal sinus/convexity, 4 (10\%) were along the base of the anterior cranial fossa/ethmoid, and $2(5 \%)$ involved the torcular herophili. Nineteen fistulas drained into a venous sinus with cortical venous reflux (Djindjian/Cognard II), 18 drained directly into a cortical vein (Djindjian/Cognard III), and 5 drained directly into an ectatic cortical vein (Djindjian/Cognard IV). ${ }^{6,7}$

Twenty-eight patients $(67 \%)$ underwent adjunctive partial embolization. There was 1 symptomatic complication after embolization: a patient harboring a cavernous fistula developed permanent abducens nerve palsy. One patient with a tentorial fistula had Onyx reflux into the internal carotid artery that remained asymptomatic yet required treatment with aspirin, and another patient with a torcular fistula sustained a small parietal lobe infarct incidentally found on MRI.

A single patient sustained an SRS-related complication (Table 1). This patient, who had had a prior intracranial hemorrhage from a convexity fistula, developed hemiparesis suspected to be an adverse effect of radiation. Sixteen patients had angiographic follow-up at a mean of 4.0 years after treatment; 13 patients $(81 \%)$ had angiographic obliteration of their dAVF. Twelve additional patients did not have angiographic follow-up but did have MRI follow-up

TABLE 1. Characteristics of and outcome parameters for an institutional cohort with dAVFs with CVD

\begin{tabular}{cccccc}
\hline Cohort & $\begin{array}{c}\text { No. of } \\
\text { dAVFs }\end{array}$ & $\begin{array}{c}\text { Angiographic } \\
\text { Occlusion }\end{array}$ & $\begin{array}{c}\text { MRI or Angiographic } \\
\text { Occlusion }\end{array}$ & $\begin{array}{c}\text { Post-SRS } \\
\text { Hemorrhage }\end{array}$ & $\begin{array}{c}\text { Symptomatic Radiation-Related } \\
\text { Complications }\end{array}$ \\
\hline All dAVFs w/ CVD & 42 & $13 / 16(81 \%)$ & $18 / 28(64 \%)$ & $1(2.4 \%)$ & $1(2.4 \%)$ \\
\hline Nonaggressive dAVFs & 19 & $5 / 6(83 \%)$ & $8 / 12(67 \%)$ & $0(0 \%)$ & $0(0 \%)$ \\
\hline Aggressive dAVFs & 23 & $8 / 10(80 \%)$ & $10 / 16(63 \%)$ & $1(4.3 \%)$ & $1(4.3 \%)$ \\
\hline
\end{tabular}


after a mean period of 3.3 years; MRI studies suggested obliteration in 5 of these patients (overall obliteration rate 64\%). Thirty-seven patients (88\%) had clinical follow-up over a mean period of 3.3 years. One hemorrhage occurred over a total of 124 patient-years of follow-up ( $0.8 \%$ annualized rate). This patient had presented with a hemorrhage and subsequently sustained a subarachnoid hemorrhage 10 months after treatment without long-term clinical sequelae.

\section{dAVF Stratified by Volume}

Forty dAVFs were stratified into 2 volumetric groups. The first 2 treated patients in our series were excluded from this stratified analysis because documentation of the exact treatment volumes was not available. Twenty dAVFs (50\%) had SRS treatment volumes $<2.0 \mathrm{~cm}^{3}$ and $20(50 \%)$ were $\geq 2.0 \mathrm{~cm}^{3}$. The mean margin dose delivered to the smallervolume group was $21.5 \mathrm{~Gy}$ over a mean volume of $1.0 \mathrm{~cm}^{3}$, whereas the larger fistulas received an average of $20.0 \mathrm{~Gy}$ to a mean volume of $4.1 \mathrm{~cm}^{3}$. In both groups, $8(62 \%)$ of 13 fistulas were obliterated radiographically (formal angiography or MRI) over a mean follow-up of 2.9 years. One patient in the larger-fistula group suffered a post-SRS hemorrhage over 68 patient-years of follow-up, whereas no patients in the smaller-fistula group had a complication or hemorrhage over 50 patient-years of follow-up.

\section{dAVF With CVD Stratified by Presentation}

Of the $42 \mathrm{dAVFs}$ with CVD at our institution, 23 (aggressive 55\%) required neurosurgical attention because of NHND or hemorrhage. The mean target volume in this group was $2.5 \mathrm{~cm}^{3}$; the mean margin dose was $21.7 \mathrm{~Gy}$. Ten patients underwent angiographic follow-up at a mean of 3.8 years after treatment, and angiographic obliteration was confirmed in 8 of the patients $(80 \%$; Table 1). Of 6 additional patients with aggressive dAVFs and only MRI follow-up, 2 had imaging studies suggesting obliteration (overall obliteration rate 63\%). Twenty patients had clinical follow-up over a mean period of 2.63 years; 1 patient sustained a radiation-related complication, and 1 patient sustained a subarachnoid hemorrhage, as noted above, over a total of 53 patient-years of follow-up for the cohort.

Nineteen patients had nonaggressive fistulas with CVD. The mean target volume was $2.8 \mathrm{~cm}^{3}$, and the mean margin dose was $20.2 \mathrm{~Gy}$. Six patients underwent angiographic follow-up at a mean of 4.4 years after treatment, and angiographic obliteration was seen in 5 cases (Table 1). Among 6 additional patients with only MRI follow-up, 3 had imaging findings suggestive of obliteration (overall obliteration rate $67 \%$ ). Seventeen patients had clinical follow-up over a mean period of 6.4 years. Over a total of 71 patient-years of follow-up, none of the patients sustained a hemorrhage or radiation-related complication. Ten (91\%) of the 11 patients who had presented with tinnitus and 4 (80\%) of 5 with ocular symptoms had improvement or resolution of symptoms after treatment.

\section{Pooled Analysis}

Of 158 records screened through our PubMed search, 37 full-text articles were assessed for eligibility, but only
TABLE 2. Pooled analysis of clinical outcomes after SRS for fistulas with CVD, stratified by presentation

\begin{tabular}{lccc}
\hline \multicolumn{1}{c}{ Parameter } & $\begin{array}{c}\text { All dAVFs } \\
\text { W/ CVD }\end{array}$ & $\begin{array}{c}\text { Nonaggressive } \\
\text { dAVFs }\end{array}$ & $\begin{array}{c}\text { Aggressive } \\
\text { dAVFs }\end{array}$ \\
\hline No. of fistulas & 197 & 124 & 73 \\
\hline $\begin{array}{l}\text { FU length in patient-years } \\
\text { Total no. of post-SRS } \\
\text { hemorrhages }\end{array}$ & $5(2.54 \%)$ & $0(0 \%)^{*}$ & $5(6.85 \%)^{*}$ \\
\hline Annual hemorrhage rate & $1.13 \%$ & $0.00 \%^{*}$ & $3.04 \%^{*}$ \\
\hline $\begin{array}{l}\text { No. of radiation-induced } \\
\text { complications }\end{array}$ & $6(3.0 \%)$ & $0(0 \%) \dagger$ & $6(8.2 \%) \dagger$ \\
\hline FU $=$ follow-up. & & & \\
${ }^{*} p=0.003$. & & & \\
$\dagger p=0.001$. & & &
\end{tabular}

6 met our eligibility requirements and were included in our analysis. ${ }^{8,14-16,18,21}$ From these 6 papers, data for 155 patients with dAVFs were extracted and combined with data on our 42 cases for a total of 197 dAVFs with CVD treated via SRS (Table 2). The mean follow-up period for this group was 2.2 years, and the rate of angiographic obliteration was 55\% among the 85 patients with radiographic follow-up. The overall annual hemorrhage rate after SRS was $1.1 \%$ (5 patients over 444.0 patient-years). None of the 124 patients with nonaggressive high-risk fistulas experienced a post-SRS hemorrhage over 279.4 patient-years; among 73 patients with aggressive dAVFs, 5 patients $(6.8 \%)$ experienced a post-SRS hemorrhage ( $\mathrm{p}$ $=0.003$; compared with nonaggressive dAVFs). The latter equates to an annual hemorrhage rate of $3.0 \%$ over 164.5 patient-years.

No complications were reported after SRS for nonaggressive dAVFs, while there was 1 permanent complication $(1.4 \%)$ and 5 transient complications $(6.8 \%, \mathrm{p}=0.001$; compared with the rate for nonaggressive dAVFs) among the patients with aggressive dAVFs. ${ }^{5,21}$ The complications included transient deterioration of clinical symptoms after SRS, followed by gradual recovery; this usually occurred in the 1st year after SRS, and 2 of these cases were attributed to thrombosis of the retrograde-draining cortical vein with resultant parenchymal edema. ${ }^{21}$

\section{Discussion}

Previous reports have demonstrated that dAVFs with CVD represent a high-risk subgroup of fistulas with a risk of causing NHND or hemorrhage. ${ }^{6,7}$ More recently, this finding has been further clarified to suggest that the aggressive subgroup of dAVFs with cortical venous refluxthat is, those that present with aggressive symptoms, such as hemorrhage and/or NHND-has a higher incidence of future hemorrhage than the subgroup with a nonaggressive presentation. ${ }^{2,19,20}$

In a natural history study, Söderman et al. calculated a 7.4\% annual incidence of repeat hemorrhage among a 32-patient cohort that had presented with hemorrhage, as compared with a $1.5 \%$ annual incidence among those without prior hemorrhage. ${ }^{19}$ Similarly, Strom et al. found a significantly lower annual rate of intracranial hemorrhage 
or NHND in patients with nonaggressive CVD compared with their aggressive counterparts $(1.4 \%$ vs $19.0 \%$, respectively; $p=0.022) .{ }^{20} \mathrm{~A}$ pooled analysis revealed a $2 \%$ annual hemorrhage rate for nonaggressive dAVFs with CVD; those presenting with NHND had an annual hemorrhage rate of $10 \%$, while those presenting with hemorrhage had an annual rebleed rate of $46 \% .^{10}$ Considering the significantly lower risk of hemorrhage for nonaggressive dAVFs with CVD, SRS may be a reasonable treatment option as the risk of hemorrhage during the latency interval prior to obliteration may be low.

We present a pooled series of 197 dAVFs treated with SRS over 444.0 patient-years of follow-up, stratifying their presenting symptoms into aggressive and nonaggressive groups. The overall angiographic obliteration rate was 55\%, which is consistent with previously reported rates following SRS for fistulas with CVD. ${ }^{4}$ Despite this obliteration rate, the posttreatment hemorrhage rate was low; across all cases, there were 5 total hemorrhages after SRS, for an annual rate of $1.1 \%$. Of these 5 reported hemorrhages, which occurred among our own institutional population and those of 2 other series, ${ }^{18,21}$ all were suffered by patients who had initially presented with aggressive symptoms prior to SRS and all occurred over approximately 164.5 patient-years of follow-up. This yielded an annual hemorrhage rate of 3.0\% in the latency period after SRS for high-risk aggressive dAVFs with CVD (compared with natural history rates of $7.4 \%-19 \%{ }^{19,20}$; this suggests some efficacy for SRS even over a follow-up period of 2.2 years-a period shorter than the typical time to obliteration.

Remarkably, we found no instances of hemorrhage after SRS for dAVFs presenting with CVD without NHND or hemorrhage over 279 patient-years of follow-up. While we remain skeptical that the actual chance of hemorrhage is 0\% following SRS for nonaggressive fistulas, this finding suggests that the annual hemorrhage rate is very low during the latency interval despite the angiographic presence of CVD. If 1 hemorrhage had occurred over the subsequent patient-year of follow-up in our pooled cohort, the annual hemorrhage rate after SRS would have been $0.6 \%$ for nonaggressive dAVFs with CVD. The number of patients with symptomatic improvement in their nonaggressive symptoms, including pulsatile tinnitus and ocular symptoms, was $14(88 \%)$ of 16 in our institutional cohort. It is also noteworthy that radiation-induced complications in the pooled cohort were low; in fact, they were significantly lower than the rate in patients with aggressive dAVFs $(\mathrm{p}=0.001)$.

\section{Study Limitations}

Although the rate of hemorrhage post-SRS was low, given the modest overall obliteration rate in our pooled analysis, a longer follow-up would be ideal to provide more rigorous and potentially more reliable clinical outcome data. Ideally, obliteration should be defined based on formal angiography, without reliance on MRI, which may not detect small or occult residuals. It is likely that the obliteration rate calculated in the pooled analysis is artificially low because of the relatively short mean follow-up in the literature. This short-term follow-up is also a limitation when attempting to draw conclusions about long-term outcomes of SRS. While care was taken to delineate the exact clinical history of specific patients from the articles used in our pooled analysis, there was significant heterogeneity among the pooled studies. For example, most studies did not provide the length of follow-up for their specific aggressive versus nonaggressive populations, and thus it had to be inferred from the overall mean follow-up data provided by the study.

Cifarelli et al. ${ }^{5}$ described 3 patients with Borden type III fistulas who suffered post-SRS hemorrhage, but they did not explicitly state whether these 3 patients had prior aggressive symptoms; thus, their data could not be reliably included in the dichotomized analysis and stratified calculations of annual hemorrhage rates following treatment with SRS. Interestingly, the majority of Borden type III fistulas in that study were initially hemorrhagic. Therefore, it is likely that the inclusion of their data in our pooled analysis would further strengthen the conclusions drawn in the present study.

Our study is also limited by its retrospective nature, allowing for treatment preferences of the surgeons involved in the care of these patients. Given that embolization or surgery is often recommended as the first-line treatment for dAVFs with CVD, it is likely that our study cohort contained preferentially more cases that failed embolization, that were believed to be poor surgical candidates, or that may have had minor hemorrhage or other characteristics suggesting a relatively low short-term bleeding risk to the treating physician; thus, our study may not have external validity for all dAVFs with CVD.

\section{Conclusions}

The rates of hemorrhage and radiation-induced complications following SRS for nonaggressive dAVFs with CVD are significantly lower than those for aggressive dAVFs. Therefore, SRS should be considered in the treatment armamentarium for nonaggressive dAVFs with CVD.

\section{References}

1. Awad IA, Little JR, Akarawi WP, Ahl J: Intracranial dural arteriovenous malformations: factors predisposing to an aggressive neurological course. J Neurosurg 72:839-850, 1990

2. Bulters DO, Mathad N, Culliford D, Millar J, Sparrow OC: The natural history of cranial dural arteriovenous fistulae with cortical venous reflux - the significance of venous ectasia. Neurosurgery 70:312-319, 2012

3. Chandra RV, Leslie-Mazwi TM, Mehta BP, Yoo AJ, Rabinov JD, Pryor JC, et al: Transarterial Onyx embolization of cranial dural arteriovenous fistulas: long-term follow-up. AJNR Am J Neuroradiol 35:1793-1797, 2014

4. Chen CJ, Lee CC, Ding D, Starke RM, Chivukula S, Yen $\mathrm{CP}$, et al: Stereotactic radiosurgery for intracranial dural arteriovenous fistulas: a systematic review. J Neurosurg 122:353-362, 2015

5. Cifarelli CP, Kaptain G, Yen CP, Schlesinger D, Sheehan JP: Gamma Knife radiosurgery for dural arteriovenous fistulas. Neurosurgery 67:1230-1235, 2010

6. Cognard C, Gobin YP, Pierot L, Bailly AL, Houdart E, Casasco A, et al: Cerebral dural arteriovenous fistulas: clinical and angiographic correlation with a revised classification of venous drainage. Radiology 194:671-680, 1995 
7. Djindjian R, Merland JJ, Théron J: Super-Selective Arteriography of the External Carotid Artery. Berlin: Springer, 1978

8. Friedman JA, Pollock BE, Nichols DA, Gorman DA, Foote RL, Stafford SL: Results of combined stereotactic radiosurgery and transarterial embolization for dural arteriovenous fistulas of the transverse and sigmoid sinuses. J Neurosurg 94:886-891, 2001

9. Gross BA, Albuquerque FC, Moon K, McDougall CG: Evolution of treatment and a detailed analysis of occlusion, recurrence, and clinical outcomes in an endovascular library of 260 dural arteriovenous fistulas. J Neurosurg 126:18841893,2017

10. Gross BA, Du R: The natural history of cerebral dural arteriovenous fistulae. Neurosurgery 71:594-603, 2012

11. Gross BA, Du R: Surgical treatment of high grade dural arteriovenous fistulae. J Clin Neurosci 20:1527-1532, 2013

12. Gross BA, Ropper AE, Popp AJ, Du R: Stereotactic radiosurgery for cerebral dural arteriovenous fistulas. Neurosurg Focus 32(5):E18, 2012

13. Liu JK, Dogan A, Ellegala DB, Carlson J, Nesbit GM, Barnwell SL, et al: The role of surgery for high-grade intracranial dural arteriovenous fistulas: importance of obliteration of venous outflow. J Neurosurg 110:913-920, 2009

14. Pan DH, Chung WY, Guo WY, Wu HM, Liu KD, Shiau CY, et al: Stereotactic radiosurgery for the treatment of dural arteriovenous fistulas involving the transverse-sigmoid sinus. J Neurosurg 96:823-829, 2002

15. Park KS, Kang DH, Park SH, Kim YS: The efficacy of gamma knife radiosurgery alone as a primary treatment for intracranial dural arteriovenous fistulas. Acta Neurochir (Wien) 158:821-828, 2016

16. Pollock BE, Nichols DA, Garrity JA, Gorman DA, Stafford SL: Stereotactic radiosurgery and particulate embolization for cavernous sinus dural arteriovenous fistulae. Neurosurgery 45:459-467, 1999

17. Rangel-Castilla L, Barber SM, Klucznik R, Diaz O: Mid and long term outcomes of dural arteriovenous fistula endovascular management with Onyx. Experience of a single tertiary center. J Neurointerv Surg 6:607-613, 2014

18. Söderman M, Edner G, Ericson K, Karlsson B, Rähn T, Ulfarsson E, et al: Gamma knife surgery for dural arteriovenous shunts: 25 years of experience. J Neurosurg 104:867875,2006

19. Söderman M, Pavic L, Edner G, Holmin S, Andersson T: Natural history of dural arteriovenous shunts. Stroke 39:1735-1739, 2008

20. Strom RG, Botros JA, Refai D, Moran CJ, Cross DT III, Chicoine MR, et al: Cranial dural arteriovenous fistulae: asymptomatic cortical venous drainage portends less aggressive clinical course. Neurosurgery 64:241-248, 2009

21. Wu HM, Pan DH, Chung WY, Guo WY, Liu KD, Shiau CY, et al: Gamma Knife surgery for the management of intracranial dural arteriovenous fistulas. J Neurosurg 105 Suppl:43-51, 2006

\section{Disclosures}

Dr. Lunsford is a stockholder of Elekta AB and has been a consultant for Insightec.

\section{Author Contributions}

Conception and design: Gross. Acquisition of data: Gross, Tonetti, Kano. Analysis and interpretation of data: Gross, Tonetti. Drafting the article: Gross, Tonetti. Critically revising the article: all authors. Reviewed submitted version of manuscript: all authors. Approved the final version of the manuscript on behalf of all authors: Gross. Statistical analysis: Gross. Administrative/technical/material support: Lunsford. Study supervision: Lunsford.

\section{Correspondence}

Bradley A. Gross: University of Pittsburgh, PA. grossb2@upmc. edu. 